\title{
Demokratie beginnt in der Nachbarschaft und endet nicht am Wahltag
}

\author{
Günter Rausch
}

Seit Jahren häufen sich die Publikationen, die in unserem Land eine „Krise der Demokratie“ beobachten (vgl. Offe 2004; Habermas 2013; Weidenfeld 2017; etc.). Auch wenn die Analysen der vielen Autor*innen zu unterschiedlichen Befunden und Erklärungen kommen, gibt es doch spätestens seit dem Einzug der rechtspopulistischen AfD in den Deutschen Bundestag im Herbst 2017 einen großen gesellschaftlichen Konsens darin, dass das Erstarken rechtspopulistischer Kräfte auch in Deutschland ein ernst zu nehmendes Signal ist, das ein „Weiter so!“ geradezu ausschließt. Am 17.3.2017 titelte Die Zeit: ,Armut gefährdet die Demokratie." Im März 2018 ließ eine Studie der Bertelsmann Stiftung aufhorchen: Demnach lebten weltweit aktuell 3,3 Milliarden Menschen unter der Herrschaft eines Autokraten. Insbesondere dort, wo soziale Spannungen nicht ausgeglichen würden, wüchsen auf Dauer antidemokratische Stimmungen: „Populistische Bewegungen und ihre Anti-Establishment-Slogans sind unter diesen Bedingungen aufgeblüht" (Bertelsmann Transformations-Index, zit. nach Bauchmüller 2018).

\section{Rechtspopulismus und soziale Deprivation}

Das „Gespenst des Populismus“ scheint die westlichen und östlichen Demokratien zu bedrohen. Offensichtlich hat der Rechtspopulismus durchaus breite Schichten der Bevölkerung im Visier. Besonders empfänglich erscheinen jene Menschen, die mit den Verwerfungen, Widersprüchen, Uneindeutigkeiten und dem Unbekannten in den entwickelten Gesellschaften schlecht umgehen können. „Die Welt ist für die Menschen voller Unsicherheiten. Rechtspopulistische Parteien sind dann eine Verlockung“ (Hauschild 2016). Unsicherheiten entstehen z. B. dort, wo Menschen von Arbeitslosigkeit betroffen oder bedroht sind, wo die Mieten kaum mehr bezahlt werden können oder gar, wie in vielen großen Städten, der Wohnungsmarkt ausgereizt ist und zahlreiche Menschen erst gar keine geeignete Wohnung finden. Neuerdings gilt es auch eine zunehmende Altersarmut zu bewältigen. Die offiziellen Armuts- und Reichtumsberichte der Bundesregierung verweisen im Übrigen auf eine stetig wachsende Zahl von Armen bei gleichzeitigem Anstieg des Reichtums in den Händen weniger Personen (vgl. Bundesministerium für Arbeit und 
Soziales 2017). Diese Diskrepanzen bergen einen sozialen Sprengstoff, der sich bei Weitem nicht nur in den Wahlergebnissen rechtspopulistischer Gruppierungen widerspiegelt. Die allermeisten der von Armut betroffenen Menschen bleiben ohnedies den Wahllokalen fern, weil sie sich von einer Wahlbeteiligung keine Besserung ihrer Lebenslage erwarten.

Der Politikwissenschaftler Wolfgang Merkel beklagte schon am 5.5.2013 unter der Überschrift „Zukunft der Demokratie - Krise? Krise!“ in der bürgerlichen Frankfurter Allgemeinen Zeitung:

„In Westeuropa gaben 1975 durchschnittlich 82 Prozent, im Jahr 2012 nur noch 72 Prozent der Wahlberechtigten in nationalen Wahlen ihre Stimme ab. In Osteuropa ist der Rückgang dramatischer: Von 72 Prozent im Jahre 1991 sank die Wahlbeteiligung 2012 auf 55 Prozent. In den Vereinigten Staaten und der Schweiz wären selbst diese Zahlen alles andere als alarmierend. [...] inzwischen deutet einiges darauf hin, dass die amerikanische Krankheit der Unterschichtsexklusion auch die europäischen und deutschen Wähler ergreift. [...] Es engagieren sich vor allem junge, gut ausgebildete Menschen für die Demokratie. [...] In den sogenannten Volksabstimmungen stimmt vor allem das besser gebildete Volk ab, die unteren Schichten fehlen meist. [...] Der Trend geht zur Zwei-Drittel- oder gar halbierten Demokratie“ (Merkel 2013).

Was hier als „Unterschichtsexklusion“ bzw. etwas zynisch als „amerikanische Krankheit" bezeichnet wurde, findet in einer Untersuchung der Bertelsmann Stiftung im September 2015 seine Bestätigung für Deutschland:

„Die Wahlbeteiligung erreicht historische Tiefstände. [...] Der typische Nichtwähler kommt aus den sozial schwächeren Milieus. [...] Demnach war die Wahlbeteiligung im September 2013 in sozial privilegierten Schichten um bis zu 40 Prozentpunkte höher als die Wahlbeteiligung in sozial schwachen Milieus. [...] Deutschland ist zu einer sozial gespaltenen Demokratie geworden. Zwischen Ober- und Unterschicht klafft eine deutliche Lücke in der Wahlbeteiligung“" (Bertelsmann Stiftung 2015).

In einer Studie zu den Bürgerschaftswahlen 2015 in Bremen findet sich bei der Bertelsmann-Stiftung ein sehr wichtiger Hinweis:

„Je prekärer die soziale Lage eines Stadtviertels, desto weniger Menschen gehen wählen. Verglichen mit den Ortsteilen, die die höchste Wahlbeteiligung verzeichneten, gehören in den Bremer Nichtwähler-Hochburgen fast zwölf Mal so viele Haushalte zu sozial schwächeren Milieus. Und dort leben nahezu vier Mal so viele Arbeitslose und doppelt so viele Menschen ohne Schulabschluss“ (ebd.).

Tatsächlich lassen sich sozial benachteiligte Milieus, z. B. die sogenannten Bildungsfernen, die Geringverdiener*innen, die Erwerbslosen, aber eben auch der Großteil der Nichtwähler*innen in allen mitteleuropäischen Städten mühelos 
räumlich verorten. Sie wohnen nicht gleichermaßen über das gesamte Stadtgebiet verteilt, sondern konzentrieren sich auf einige wenig attraktive Stadtbezirke, zumeist in den Randbereichen oder in mehrfach belasteten Wohngebieten.

Didier Eribon beobachtet allerdings darüber hinaus, wie sich ,große Teile der prekarisierten und verwundbaren Unterschicht mit Leuten aus Handelsberufen, mit wohlhabenden [...] Rentnern, sogar mit faschistischen Exmilitärs und traditionalistischen Katholiken verbinden" (Eribon 2016: 128). Tatsächlich sind Phänomene wie Ängste vor Unbekannten, Abgrenzungen gegenüber Ungewohntem, Fremdenfeindlichkeit, Nationalismus etc. durchaus in breiten Bevölkerungsschichten vorzufinden. Die Gründe hierfür sind vielschichtig, oftmals aber in den biografischen Bildungs- und Sozialisationsgeschichten und deren Sozialräumen verwurzelt. „Das Viertel, in dem man lebt, ist für das Selbstverständnis und die Sicht auf die Welt nun wichtiger als der Arbeitsplatz und die Position im sozialen Gefüge“" (ebd.: 140).

Was Eribon „Viertel“ nennt, wurde bei uns lange Zeit als Wohngebiet, Stadtteil oder mancherorts auch Kiez bezeichnet. Inzwischen haben sich hierfür auch die Begriffe „Quartier“ oder gar „Nachbarschaft“ herausgebildet. Letzteres knüpft an den anglo-amerikanischen Terminus der ,neighborhoods“ an, wie er in den Lehrbüchern der Sozialwissenschaften, insbesondere in historischen Einführungen, seit Langem verwendet wird. Der nachfolgende Exkurs soll einerseits zeigen, dass die Probleme, mit denen wir heute befasst sind, keineswegs neu sind, und dass es andererseits durchaus bewährte Handlungskonzepte gibt, deren Transformationsperspektiven zu überprüfen wären.

\section{Ein Exkurs: traditionelle Ansätze in der Settlement- und Demokratiebewegung}

Seit 1884 gab es im Osten von London erste „Settlements“. Das Pastorenehepaar Samuel und Henrietta Barnett zog mit Studierenden und anderen sozial Motivierten in eines der Elendsquartiere der britischen Hauptstadt. Sie teilten den Alltag und versuchten, vertrauensvolle Beziehungen zu den dort lebenden armen Menschen aufzubauen. In Kooperation mit der Universität sollten u. a. die Studierenden dort zwei Jahre leben, damit sie die Lebensbedingungen der Slumbewohner*innen aus erster Hand kennen lernten. Letztlich sollten die Lebensbedingungen der Bewohner*innen so verändert werden, dass sie ein Leben würden führen können, wie es den Gepflogenheiten der damaligen Gesellschaft entsprach. Dementsprechend gab es verschiedene Angebote, z. B. Abendkurse, kulturelle Veranstaltungen sowie Diskussions- und Jugendclubs. Das war nicht immer einfach: 


\begin{abstract}
„Das Gruppenleben musste ebenso geübt und ausgehalten werden wie die Mitwirkung in den Clubs und Vereinen im Wohngebiet von Toynbee Hall. Der tägliche Umgang der Residents untereinander und mit den Menschen, die sie draußen antrafen und um sich versammelten, das Clubleben und die Arbeitsgemeinschaften verlangten eine soziale Gruppenarbeit“ (Wendt 1990: 150 f.).
\end{abstract}

Ein deutscher zeitgenössischer Beobachter, der im Jahre 1913 mehrere Settlements besuchte, bemerkte: „Toynbee Hall ist, um es einseitig auszudrücken, zum ,politischen Settlement' geworden. Es ist mehr interessiert an Fragen des öffentlichen Lebens als am Leben des einzelnen Menschen“ (Picht 1913: 113 f.).

Recht schnell griffen die Ideen der Settlement-Bewegung auf Amerika über. Bereits 1886 wurde in New York die „Neighbourhood guild“ gegründet. Deren Leitgedanke war, ,die Hilfe nicht wie seither von oben nach unten in der wohlbekannten Haltung des Wohltäters zu bringen, sondern dem leidenden Volke Freund zu werden und unter den Ärmsten und Elendesten Nachbar unter Nachbarn zu werden“" (Münsterberg 1906: 100). In einem der großen Slums Chicagos entstand unter der Leitung von Jane Addams das Hull House:

„Es ist die Aufgabe von Hull House ein Zentrum für ein höheres, öffentliches und soziales Leben zu sein; Einrichtungen der Bildung und der Philanthropie zu gründen und zu unterhalten und die Lebensbedingungen in den industriellen Bezirken von Chicago zu untersuchen und zu verbessern“ (Addams, zit. in Müller 1999: 73).

V. a. Jane Addams arbeitete unermüdlich auf lokaler, nationaler und internationaler Ebene in unterschiedlichsten Gremien und Initiativen, um Veränderungen zugunsten der armen und benachteiligten Menschen herbeizuführen. Wendt formulierte das so:

„Die Residents erkannten bald, dass ihre Aktivitäten erst dann eine dauernde Veränderung und Verbesserung des Lebens im Wohngebiet bedeuten konnten, wenn sie sich in der kommunalen Politik fortsetzten. Die ,settlement workers' mischten sich ein, beteiligten sich an der lokalen Selbstverwaltung und ermunterten andere Bewohner zu politischem Handeln“ (Wendt 1990: 151).

Dabei stand das gemeinschaftliche Handeln im Mittelpunkt der Anstrengungen zur Überwindung sozialer Notlagen und Probleme. Die Menschen strebten dabei eine gleichberechtigte, symmetrische Ebene an: „Wer in ein Settlement zog, erwarb keinerlei Macht per Entscheidung über eine Mittelvergabe, keine Gewalt, die ihn stützen oder schützen konnte“ (ebd.: 150).

Diese Residents (vergleichbar vielleicht mit den heutigen „Gemeinwesenarbeiter*innen“) gingen „,von der ungebrochenen Überzeugung aus, dass Menschen fähig sind, ihr Leben selbst zu bestimmen und zu gestalten, wenn sie daran nicht 
zwanghaft durch die sozialen Umstände gehindert werden, unter denen sie leiden“ (Müller 1988: 94). Bei Jane Addams ist darüber hinaus bereits der Gedanke von sozialer Gerechtigkeit mit einer „universalen Solidarität“" gekoppelt:

„Wenn Solidarität der menschlichen Interessen verwirklicht werden soll, wird es undenkbar, dass eine Klasse von Menschen für die vermeintlichen Bedürfnisse einer anderen Klasse von Menschen geopfert werden soll. [...] Für verschiedenste Gruppen von Männern und Frauen in der ganzen Welt ist offenbar die Zeit gekommen, um sicherzustellen, dass alle Menschen gegen den Hungertod versichert werden müssen“" (Addams 1947, zit. nach Staub-Bernasconi 1995: 5).

Es ist bemerkenswert, wie Barnett, Addams und viele andere fortschrittliche Bürger*innen des 19. Jahrhunderts die „soziale Frage“, also die Frage nach der Überwindung der Massenarmut durch die Herstellung von Gerechtigkeit, mit den Vorstellungen eines demokratischen Umgangs miteinander im Kleinen wie in der großen Gesellschaft („Great Community“) verbanden. Diese Verbindung der sozialen Lebensverhältnisse mit demokratiepolitischen Perspektiven hat der US-amerikanische Pädagoge und Philosoph John Dewey (1859-1952) nicht nur philosophisch beleuchtet, sondern ganz konkret auf das alltägliche Leben heruntergebrochen. Für ihn ist Demokratie weniger eine Frage der Regierungsform, als eine Form des miteinander Umgehens. Dementsprechend beginnt für ihn Demokratie keineswegs bei den Parlamentswahlen oder dergleichen, sondern im alltäglichen Zusammenleben: „Democracy must begin at home and its home is the neighborly community" (Dewey 1927: 368). Interessant ist an dieser Aussage nicht nur der Verweis auf das Zuhause, das persönliche Heim oder die Wohnung, sondern die Hinwendung zur nachbarschaftlichen Gemeinschaft. Diese Gemeinschaft gelte es face-toface über persönliche Beziehungen und durch das gemeinschaftliche Handeln zu entwickeln.

„Wo immer es eine gemeinsame Tätigkeit gibt, deren Folgen von jedem einzelnen der an ihr teilnehmenden Personen für gut befunden werden, und wo die Verwirklichung des Guten von der Art ist, dass sie ein tatkräftiges Verlangen und Bemühen hervorruft, es zu erhalten, weil es ein von allen geteiltes Gut ist, da gibt es insofern eine Gemeinschaft" (Dewey 1988: 328).

Dewey verwies auf das gemeinsame Denken, das miteinander Sprechen und schließlich auf die Zusammenarbeit. In diesen Aushandlungsprozessen entwickelten sich nicht nur Gemeinschaften, sondern zugleich auch die Menschen. Insoweit lebt Demokratie dann auch von Bildungs- und Erziehungsprozessen, die bei Dewey nicht das Pauken von vorgefertigten Lernprogrammen meinen, sondern nur das alltagsnahe Gemeinschaftslernen auf der Basis des von ihm so bezeichneten Learning by Doing“. Knoll spricht deshalb von einem Dreiklang von Kommuni- 
kation, Partizipation und Kooperation als unerlässliche Voraussetzung, ,damit die Menschen zum einen ihre Potentiale ausschöpfen und sich voll verwirklichen konnten; zum anderen seien sie dann auch in der Lage, ihre unveräußerlichen Rechte auf Selbstbestimmung, Chancengleichheit und soziale Gerechtigkeit uneingeschränkt wahrzunehmen“" (Knoll 2018).

Inwiefern eignen sich diese hier skizzierten historischen Handlungskonzepte, noch dazu aus der anglo-amerikanischen Geschichte, zur Bewältigung der eingangs zitierten „Krise der Demokratie“ im 21. Jahrhundert in Deutschland? Zur Beantwortung dieser Frage soll beispielhaft auf langjährige Erfahrungen in einer Hochhaussiedlung in Freiburg im Breisgau zurückgegriffen werden, in der seit 1989 mittels Gemeinwesenarbeit im Rahmen einer Bewohnerinitiative (,Forum Weingarten") immer wieder versucht wird, exemplarisch Lernprozesse zu einem solidarischen und demokratischen Miteinander zu entwickeln.

\section{Gemeinwesenarbeit am Beispiel der Hochhaussiedlung Freiburg- Weingarten}

Weingarten ist eine typische Hochhaussiedlung der 1960er-Jahre, die v. a. durch den sozialen Wohnungsbau jener Zeit geprägt ist. Heute leben im Kerngebiet rund 10.000 Menschen, von denen ein auffallend hoher Teil einen Migrationshintergrund aufweist, über ein unterdurchschnittliches Einkommen verfügt, eher selten eine Hochschule besuchen konnte und verhältnismäßig wenig zur Wahl geht. Schon wenige Jahre nach dem Erstbezug machte dieser Stadtteil in der Öffentlichkeit zunehmend negative Schlagzeilen. „Sozialer Brennpunkt“ war die landläufige Bezeichnung jener Zeit. Als die Probleme immer drängender wurden, fanden sich Bewohner*innen, Kirchengemeinden und Stadtteilgruppen zu einer neuen Bürgerinitiative zusammen. Ziel war es, über ein neu zu entwickelndes Bewohnerengagement diese Entwicklungen zu stoppen und wieder einen ,liebens- und lebenswerten Stadtteil“" zu schaffen. Engagiert waren zu diesem Zeitpunkt vor allem Vertreter*innen der bürgerlichen Mittelschicht, die in diesem Stadtteil deutlich in der Minderheit waren. Die schweigenden oder als passiv empfundenen Mehrheitsangehörigen sollten ,aktiviert“" werden. Deshalb heißt es in der Gründungssatzung des Trägervereins:

„Der Verein und seine Einrichtungen sollen eine konsequent bewohnerorientierte Arbeitsweise gewährleisten [...]. [Es wird] erwartet, dass dadurch eine möglichst große Anzahl engagierter Bürger des Stadtteils ihre spezifischen Erfahrungen, Wünsche und Möglichkeiten im Rahmen einer Mitarbeit zum Allgemeinwohl Weingartens und seiner Bewohner einbringen kann“ (Forum Weingarten 1989). 
Zu diesem Zweck der Aktivierung erfolgte zunächst die Anmietung eines leer stehenden Ladengeschäftes (Stadtteilbüro) als Anlaufstelle inmitten eines Einkaufszentrums sowie die Anstellung des ersten Gemeinwesenarbeiters. Als Arbeitsprinzipien wurden in der Konzeption des Forums Weingarten (ebd.) eine „konsequente Alltags- und Lebensweltorientierung" gefordert. Der Gemeinwesenarbeiter sollte von Beginn an das Engagement der Bewohner*innen herausfordern und fördern. Es galt, ,,aus Betroffenen Beteiligte werden“ zu lassen. Methodisch und didaktisch fand eine Anlehnung an das Community Organizing im Sinne von Saul Alinsky (1941) sowie an die „Pädagogik der Unterdrückten“ nach Paulo Freire (1973) statt. Von Alinsky wurde insbesondere die konsequente Gehstruktur (immer wieder Haustüraktionen im Sinne von Türklinkenputzen), das Anknüpfen an die „Issues“, also die brennenden Probleme der Menschen, sowie der gezielte Aufbau von eigenständigen demokratischen Bewohnerstrukturen (Arbeitskreise, Sprecherräte, Bewohnerversammlungen etc.) übernommen.

Die Betroffenen sollten als die Expert*innen ihrer Lebensverhältnisse anerkannt und wertgeschätzt werden. Im Sinne des Empowerments galt es, an den jeweiligen Interessen und Stärken anzuknüpfen und stets die partnerschaftliche $\mathrm{Zu}$ sammenarbeit auf der Ebene des Dialoges zu suchen. Letztere meinte v. a. die Begegnung auf Augenhöhe und einen Verzicht auf Vordenkertum, auf alle Facetten der Pseudobeteiligung oder stellvertretendes Handeln. Es mussten „Freiräume“ oder gar „Leerräume“ angeboten und freigehalten werden. So wurde beispielsweise der angemietete Laden eine ganze Weile nicht möbliert. Er blieb leer. Mit Bierbänken und -tischen wurde improvisiert und auch auf diese Weise allen deutlich gemacht, dass sie selber aufgefordert waren, mitzugestalten und mitzuentscheiden! Wer Menschen beteiligen will, die ihr Leben lang gelernt haben, dass alle wichtigen Entscheidungen, die sie betreffen, über ihre Köpfe hinweg getroffen werden, sollte ihnen die Möglichkeit bieten, eigene, neue Erfahrungen der Selbstwirksamkeit zu machen. Geduldig müssen diese Menschen erfahren können, dass die Einladung zum Mitdenken und Mitentscheiden ernst gemeint ist. Und sie müssen erleben, dass ihr persönliches Engagement wichtig und wirksam ist.

Paulo Freire, der sich in den 1950er- und 1960er-Jahren des vergangenen Jahrhunderts mit der Apathie und der Resignation von armen lateinamerikanischen Menschen befasste, hat Bedingungen für die Überwindung des Rückzuges benachteiligter Bevölkerungsgruppen genannt:

„Weil Dialog Begegnung zwischen Menschen ist, darf er keine Situation bilden, in der einige Menschen auf Kosten anderer die Welt benennen. Vielmehr ist er ein Akt der Schöpfung. Er darf nicht als handliches Instrument zur Beherrschung von Menschen durch andere dienen" (Freire 1973: 72). 
Dies erfordert ein neues „Expertenprofil““ für Professionelle. Die besonderen Fähigkeiten des wissenschaftlich qualifizierten Fachmannes sollen in diesen Kontexten nicht in eine eloquente Performance bzw. Selbstprofilierung der Sozialarbeitenden münden, sondern in eine hohe Sensibilität für die Wahrnehmung der Fähigkeiten, Neigungen und Interessen, aber auch der Sorgen, Nöte und Ängste der Bewohner*innen. Diese Grundregeln sind aus der humanistischen Psychologie und den abgeleiteten Gesprächsführungsmodellen längst bekannt: Vertrauen schaffen, geduldig zuhören, einfühlsames Nachfragen, aber auch positives Verstärken, freundliches Ermutigen, reflektiertes Verstehen etc.

In der Praxis ist weniger der Druck von außen, z. B. die Notwendigkeit vorzeigbarer Erfolge das Problem, es sind vielmehr die Erwartungshaltung und die Einstellungen der Betroffenen. In deren bisherigen Lebenserfahrungen waren es stets die akademisch profilierten, versierten Leute oder die Vertreter*innen der Ämter und Behörden, die an ihrer Stelle gedacht und gehandelt haben. Im Laufe ihres Lebens haben sie für sich gelernt, dass ,die da oben“ ja doch machen, was sie wollen, und ihre Meinung nicht wirklich gefragt ist. Sie ziehen es deshalb meist vor, zu schweigen, bestenfalls zu schimpfen und sich herauszuhalten. Der Theologe Bonhoeffer, der seine Beteiligung am Widerstand gegen die Nazidiktatur mit dem Leben bezahlen musste, schrieb noch kurz vor seiner Ermordung über das Schweigen der Massen während des Faschismus:

„Die Macht der einen braucht die Dummheit der anderen. Der Vorgang ist dabei nicht der, dass bestimmte - also etwa intellektuelle - Anlagen des Menschen plötzlich verkümmern oder ausfallen, sondern dass unter dem überwältigenden Eindruck der Machtentfaltung dem Menschen seine innere Selbständigkeit geraubt wird und dass dieser nun - mehr oder weniger unbewusst - darauf verzichtet, zu den sich ergebenden Lebenslagen ein eigenes Verhalten zu finden“ (Bonhoeffer 1985: 14 f.).

Er mahnte außerdem, „dass nicht ein Akt der Belehrung, sondern allein ein Akt der Befreiung die Dummheit überwinden könnte“ (ebd.).

Befreiung kann hier durchaus im Sinne Kants verstanden werden, also als der „Ausgang des Menschen aus seiner selbst verschuldeten Unmündigkeit“" (Kant 1784: 481). Dieser Gedanke, dass sozial benachteiligte Menschen nicht einfach willenlose, ohnmächtige Objekte eines übermächtigen Schicksals sind, passt nicht unbedingt in sozialromantische Mitleidsmodelle. Die Betroffenen müssen lernen, was sie selbst leisten können, und dass sie mit ihrer Eigeninitiative die maßgeblichen Leistungen ihrer Emanzipation selbst erbringen müssen. Gemeinsam mit anderen müssen sie die Erzählfäden ihres Lebens aufgreifen und an ihrer Lebensgeschichte stricken, die freilich immer auch eine gesellschaftliche Konstruktion ist. V. a. braucht es Räume und Gelegenheiten der Begegnung und des Einübens von gemeinsamem Tun. Das Quartier bietet vielfältige Möglichkeiten der Betätigung 
und Entfaltung. Es ist nicht nur Behinderungsraum, sondern auch Lernfeld und Möglichkeitsraum. Von allein geht dies allerdings nicht: „Wer dauerhaft Objekt von Entscheidungen gewesen ist, kann nicht ohne Unterstützung Subjekt eigener Entscheidungen werden. Kurz: Man braucht Lern- und Handlungsinitiatoren“ (Gronemeyer \& Bahr 1977: 202).

Um den Teufelskreis von Benachteiligung, Rückzug und Resignation zu durchbrechen, wurde in Weingarten mithilfe der Gemeinwesenarbeit an die Stelle vordefinierter Ziele, ausgetüftelter Strategien und von Fachleuten entworfenen Entwicklungskonzepten ein schlichtes Modell der Alltags- und Lebensweltorientierung mit einem stringenten Ansatz des Empowerments angewandt. Die Betroffenen sollten selbst sagen, wo der Schuh drückt und wie es anders und besser werden sollte. Über Haustürgespräche wurden die Kontakte hergestellt, erste Beziehungen geknüpft und die Einladungen zu überschaubaren Hausversammlungen, Themenabenden etc. ausgesprochen.

\section{Partizipation als Entwicklungskonzept}

Soweit zur Vorgeschichte eines Partizipationsmodells, das vor bald 30 Jahren eher mit Experimentcharakter begonnen hatte. Das Forum Weingarten gibt es u. a. mit seinem Stadtteilbüro heute noch. Eine wissenschaftlich fundierte Evaluation der langjährigen Arbeit des Forums Weingarten liegt bis heute nicht vor. Es gibt jedoch eine Vielzahl von Publikationen und kleineren Forschungsprojekten sowie etliche wissenschaftliche Arbeiten, darunter zwei Dissertationen, die sich u. a. mit diesem Partizipationsansatz in Weingarten auseinandersetzen.

\subsection{Beteiligen - aber wie?}

Annette Brox, die seit 1994 Gemeinwesenarbeiterin und Geschäftsführerin des Forums Weingarten ist, schrieb in einem Fachaufsatz mit dem Titel „Bewohnerbeteiligung ernst gemacht“:

„Punktuelle, formalisierte Beteiligungsverfahren im Rahmen der gesetzlichen Vorgaben reichen hierfür längst nicht aus. Beteiligung muss ernst gemeint, niedrigschwellig, transparent und kontinuierlich sein. Aufgabe der Gemeinwesenarbeit ist es, geeignete Beteiligungsstrukturen aufzubauen, gemeinsam mit den BewohnerInnen politisch einzufordern und die Selbstorganisation der BewohnerInnen zu unterstützen“" (Brox 2012: 25).

In Weingarten gibt es deshalb z. B. eine gewählte Bewohnervertretung, die in Sanierungs- und Stadtteilgremien die Betroffenen vertritt. Alle zwei Jahre werden 
ordentliche Wahlen durchgeführt, bei der 16 Bewohnervertreter*innen gewählt werden. Alle Mieter*innen sind aufgefordert, zu kandidieren und so auch Verantwortung für das Quartier zu übernehmen. Um eine hohe Wahlbeteiligung zu erreichen, werden die versiegelten Urnen von Tür zu Tür getragen. Anja Bochtler berichtete in der Badischen Zeitung am 25.10.2011:

„Wer hier mitmischt, will sich engagieren - auch für andere. Darum sind 20 ehrenamtliche Wahlhelferinnen und -helfer zwei Wochen lang bei 2146 Wohnungen vorbei gegangen, deren Mieter wahlberechtigt waren. Oft haben sie mehrmals geklingelt. Viele Wahlhelfer haben selbst kandidiert, einige sind seit Jahren bekannt. Das zahlt sich aus, wie sich zeigt, als die Quartiersarbeiter die Ergebnisse präsentieren: Die Beteiligung ist da am höchsten, wo viele Kandidaten wohnen und aktiv sind - etwa in der Bugginger Straße 46 in Weingarten-West, wo 80 Prozent der Mieter gewählt haben, oder in der Krozinger Straße 4 in Weingarten-Ost, wo die Beteiligung bei 78 Prozent lag" (Bochtler 2011).

Die Wahlbeteiligung bei den Gemeinderatswahlen 2014 lag dagegen bei 30,9\% und bei den stets besser besuchten Bundestagswahlen (hier: 2013) bei 58,6\%. Im ersten Wahlgang der Oberbürgermeisterwahlen 2018 gingen nur noch $27 \%$ der Weingartener*innen wählen (vgl. Stadt Freiburg 2018).

Der eigentliche Erfolg dieser Arbeit ist derzeit offensichtlich nicht bei der Wahlbeteiligung nachzuweisen. Dagegen ist es inzwischen über einen sehr langen Zeitraum hinweg gelungen, eine eigenständige demokratische Interessenvertretung in einem sozial benachteiligten Quartier zu etablieren, in dem Betroffene Verantwortung für sich und die Gemeinschaft übernehmen. Einige wenige Personen sind von Anfang an dabei; manche sind inzwischen verstorben, andere aus unterschiedlichen Gründen ausgeschieden. Immer wieder gelingt es aber den ehrenund den hauptamtlichen Akteur*innen neue Mitstreiter*innen zu finden.

Im gegebenen Partizipationskontext erscheint dieses Beteiligungsmodell umso bedeutsamer, als dieses selbstorganisierte Gremium seit vielen Jahren auch gegenüber Politik und Verwaltung die Bewohner*innen vertritt und als kompetenter Kooperationspartner agiert.

\subsection{Konflikte im nachbarschaftsorientierten Demokratiemodell}

Deweys Idee, dass Demokratie in der Nachbarschaft beginne, stand durchaus in diesem konkreten Beteiligungsmodell Pate. Es ist wohl bislang nur in Freiburg im Breisgau über einen längeren Zeitraum hinweg praktiziert worden. Daneben wurden weitere strukturelle Mitsprachekonzepte der Mieter*innen entwickelt, z. B. demokratisch gewählte Mieter- und Sprecherräte. Durch ein niedrigschwelliges Verfahren wird eine Wahlbeteiligung von 70 bis $80 \%$ erreicht. V. a. können im- 
mer wieder Menschen dafür gewonnen werden, gemeinsam mit anderen Bewohner*innen Verantwortung im und für das Quartier wahrzunehmen. Die gewählten Vertreter*innen treffen sich formell und informell, um sich über Themen und Probleme in der Nachbarschaft auszutauschen und gemeinsam nach Lösungswegen zu suchen. Konflikte bleiben dabei nicht aus, zumal es sich um ein Wohngebiet handelt, in dem nicht nur sehr viele Arbeitslose, Ausländer*innen, Arme, Alleinerziehende und Alte leben, sondern tatsächlich eine Vielheit von Kulturen, Lebenswelten und Lebensgeschichten zusammenkommen. Hier sind Mediationen oder Klärungsgespräche in Konfliktfällen, insbesondere durch die Hauptamtlichen, nicht selten. Aber zumeist lassen sich die Dinge im Gespräch klären.

Als weiteres Beispiel soll ein seit 1991 erprobtes Mitwirkungsmodell der Mieter*innen bei der Neubelegung von Wohnungen genannt werden: Wird eine Wohnung frei, so stellen sich die Bewerber*innen den künftigen Nachbar*innen bei einem gemeinsamen, vom Gemeinwesenarbeiter bzw. von der Gemeinwesenarbeiterin moderierten Treffen vor.

Hierzu werden alle Mieter*innen des betroffenen Stockwerkes sowie des Stockwerkes darüber und darunter eingeladen. Das Treffen findet zumeist in den Gemeinschaftswohnungen im Erdgeschoß der Hochhäuser statt. Nach der Vorstellung der Bewerber*innen beraten und beschließen die Nachbar*innen, wer wohl am besten in ihr Haus oder auf ihr Stockwerk passt. In diesem Modell können die Mieter*innen nicht nur mitentscheiden, wer künftig in der Nachbarschaft wohnen wird, vielmehr übernehmen sie zugleich auch Verantwortung für ein soziales Miteinander. Damit sollen nicht zuletzt die Grundlagen für gelingende Nachbarschaften und Begegnungen geschaffen werden. Während sich für gewöhnlich die neuen Nachbar*innen sich mehr oder weniger zufällig erst nach einer gewissen Zeit irgendwie kennenlernen, wird hier die Gelegenheit geschaffen, sich schon vor dem Einzug zu „beschnuppern“, etwas voneinander zu erfahren und v. a. auch gewisse Gepflogenheiten und Vereinbarungen schon kennenzulernen, bevor man eingezogen ist.

Ein solches Beteiligungsverfahren birgt naturgemäß vielfältige Konflikte und Reibungsflächen zwischen Vermietungsgesellschaft und den mitbestimmenden Mieter*innen, aber auch zwischen einzelnen Mietparteien und Interessent*innen. Von Anfang an gab es Spannungen zwischen der Verwaltung der städtischen Wohnbaugesellschaft und dem Forum Weingarten. Aus der Sicht der Verwaltung war das gesamte Bewerbungsverfahren nunmehr deutlich erschwert und langwierig. Aus der Stadtteilperspektive gab es immer wieder Klagen, dass zu wenige geeignete Bewerber*innen vorgestellt würden. Hinzu kamen immer wieder Kommunikations- und Verständigungsprobleme mit wechselseitiger Schuldzuweisung. Als die Stadtbau GmbH schließlich im Jahre 2010 die Zusammenarbeit aufkündigte, entschied sich eine Gruppe von Studierenden unter wissenschaftlicher Lei- 
tung des Autors, dieses Mitbestimmungsverfahren einmal kritisch unter die Lupe zu nehmen. Es wurden im Studienjahr 2010/2011 Interviews mit Expert*innen geführt, relevante Sitzungen teilnehmend beobachtet und die Akten sowie das Archivmaterial ausgewertet. Schließlich und nicht zuletzt wurde in einem Bezirk eine ausführliche Haustürbefragung von 114 Bewohner*innen mittels leitfadengestützter Interviews durchgeführt. Eine der wichtigsten Erkenntnisse dieser Untersuchung war sicherlich, dass ca. $70 \%$ der 114 Befragten das Verfahren als ,(eher) sinnvoll“" erachteten, wobei jene Mieter*innen, die die Einführung des Verfahrens vor 15 Jahren selbst miterlebt hatten, dieses tendenziell besser bewerteten. Nahezu alle Befragten (96\%) gaben an, dieses Verfahren zu kennen, und immer noch sehr viele $(84 \%)$ wollen mindestens einmal daran teilgenommen haben. Danach befragt, wieso sie daran teilgenommen hätten, antworteten einige z. B.: ,aus Interesse an den Bewerbern“, „,um sich ein Bild der Personen machen“ oder „,zur Entschärfung der sozialen Lage“. Es gab aber auch kritische Einwürfe, z. B.: „will nicht über andere bestimmen“, „,möchte nicht die Macht über Wohnungsvergabe haben“ oder „das Verfahren hat keinen Sinn“. Die Sinnfrage war explizit gestellt worden, worauf $70 \%$ der Befragten das Verfahren als eher sinnvoll empfanden. Rund $60 \%$ stimmten der Aussage zu, dass das Verfahren dazu beigetragen habe, „dass die Stimmung im Haus eher gut ist“. Die insgesamt positiven Ergebnisse dieser Untersuchung, die leider nie schriftlich veröffentlicht worden sind, wurden auf einer Versammlung unter Anwesenheit der Stadtbau GmbH, der Gemeinwesenarbeiter*innen, einiger Gemeinderäte und der Presse auch den Bewohner*innen vorgestellt.

Vielleicht war das Timing zufällig passend, denn wenige Tage nach dieser Präsentation kam es zu einem versöhnlichen Agreement zwischen den Akteur*innen. Man verständigte sich auf einige Korrekturen im Verfahren und arbeitete nachfolgend mit modifizierten Regeln weiter. Aber auch diese damalige Einigung war nur eine vorübergehende. Immer wieder kam es zu mehr oder weniger deutlichen Unstimmigkeiten, die allerdings im Jahre 2017 erheblich eskalierten. Es kann an dieser Stelle unmöglich die Komplexität dieser Vorgänge dargestellt und analysiert werden. Offensichtlich war es zumindest in einem Hochhaus, das in diesem Beteiligungsmodell von Anfang an involviert war, im Laufe der Jahre zwischen den Bewohner*innen zu gewissen „Blockbildungen“ bzw. „Einflussgruppierungen" gekommen, die den Eindruck erwecken mussten, dass bei den Voten der Bewohner*innen nicht immer nach den Grundsätzen von Fairness und Chancengleichheit gehandelt wurde. Letztendlich entschied deshalb die Leitung der städtischen Wohnbaugesellschaft, für dieses Hochhaus das Mitwirkungsmodell bis auf Weiteres auszusetzen. Nach vielen Gesprächen einigten sich dann doch alle Beteiligten wieder darauf, den begonnenen Weg dieses Beteiligungsmodells, mit Ausnahme des einen Hochhauses, fortzusetzen. 
Im Gespräch mit den Gemeinwesenarbeiter*innen und auch mit den Bewohner*innen wurde aber auch deutlich, wie schwierig es ist, die vielen unterschiedlichen Einstellungen, Sichtweisen, Lebensstile, Weltanschauungen, Lebenswelten usw., die es nun einmal in einer Großwohnsiedlung mit Menschen aus über 100 Nationen gibt, unter einen Hut zu kriegen. Das beginnt bei der persönlichen Einladung zu einem Auswahlverfahren und endet noch lange nicht bei der Moderation der Vorstellungsgespräche und Bewohnerentscheide. Und ganz sicherlich warten die Menschen auch nicht schon darauf, wieder einmal an einem solchen Mitbestimmungstreffen teilnehmen zu können. Eine der Öffentlichkeit zugängliche, kritische Reflexion und Evaluation der verantwortlichen Rolle der professionellen Begleiter*innen dieses Verfahrens liegt leider nicht vor.

\subsection{Wirksamkeit im Hinblick auf das Wahlverhalten}

Des Weiteren soll als ein zusätzlicher kritischer Aspekt im Hinblick auf die Wirksamkeit der Quartiersarbeit im Demokratisierungsprozess vor Ort das Wahlverhalten der Bewohner*innen betrachtet werden. Auch hier lassen sich ambivalente Befunde diskutieren, insbesondere die genannten niedrigen Wahlbeteiligungsquoten trotz langjähriger, intensiver sozialer Stadtteilarbeit. Hierfür gibt es keine überzeugenden Erklärungsmodelle. Empirische Untersuchungen vor Ort gibt es hierfür gar keine. Dagegen werden diverse Hypothesen diskutiert. Einerseits ist es zweifelsfrei, dass die ,aktivierten“ Bürger*innen, die zum Sprecherrat etc. zählen, allesamt von ihrem Wahlrecht Gebrauch machen. Mitunter engagieren sie sich auch in Parteien oder politischen Initiativen im Quartier. Warum aber haben all die vielfältigen Aktivitäten über die Jahre hinweg verhältnismäßig wenig Strahlkraft im öffentlichen Raum? Liegt es daran, dass die individuellen biografischen Lebenserfahrungen sowie die allgemeinen gesellschaftlichen Rahmenbedingungen für die Lebensbewältigung in der Wahrnehmung der von Armut und Ausgrenzung bedrohten Menschen so signifikant entmutigend und enttäuschend sind, dass sie trotz manch erlebter positiver Kontrollerfahrung bzw. Selbstwirksamkeit nach wie vor kein Vertrauen in die parlamentarischen Vertreter*innen und ihre Organe haben? Es gibt allerdings ein bedeutsames Ereignis: Als im Jahre 2006 die Spitze der Stadtverwaltung alle städtischen Wohnungen verkaufen wollte, gab es in der Stadt Freiburg über Monate hinweg eine auch bundesweit beobachtete Protestbewegung, die auch auf die Aktivierung der betroffenen Mieter*innen zielte. Schlussendlich gab es ein erfolgreiches Bürgerbegehren mit abschließendem Bürgerentscheid.

Bei dieser Abstimmung war es so, dass die Wahlbeteiligung im Stadtteil Weingarten mit 49,2 \% fast zehn Prozentpunkte über der gesamtstädtischen Quote lag. Es gab hierfür zwei Erklärungsansätze: einerseits, dass es hier um die eigene 
Wohnung ging, also um eine Angelegenheit mit einer hohen persönlichen Betroffenheit. Viele befürchteten, dass nach einer Privatisierung die Mieten deutlich ansteigen würden und/oder die Instandhaltung der Wohnungen vernachlässigt würde. Andererseits bot der Bürgerentscheid eine klare Chance, durch das eigene Handeln diese Bedrohungen abzuwehren. Das Kreuzchen am Wahlschein hatte dieses Mal mehr oder weniger direkte Auswirkungen auf die eigene Lebenslage. Von daher entsprach es einer gewissen Plausibilität, trotz allgemeiner ,Wahlverdrossenheit" dieses Mal doch zu den Urnen zu gehen. Klus, der in seiner Dissertation diesen Freiburger Bürgerentscheid wissenschaftlich analysiert hat, kommt zu folgendem Schluss:

„Offensichtlich wurden dort hohe Beteiligungsquoten erzielt, wo Stadtteil- und Mietergruppen der Bürgerinitiative ,Wohnen ist Menschenrecht" besonders aktiv waren und in ihren direkten Aktionsformen auf die Aktivierung der Betroffenen abzielten. [...] Zudem wird auf eine aktive Rolle von Einrichtungen der Gemeinwesenarbeit bzw. der Quartiersarbeit bei der Mobilisierung der MieterInnen in einigen Stadtteilen und insbesondere in Freiburg-Weingarten verwiesen“" Klus 2013: 215).

Es stellt sich die Frage, wie das Wählerverhalten hinsichtlich der Stimmenanteile für rechtspopulistische Parteien aussah: Die Landtagswahlen im Jahre 2016 erbrachten für den Stadtteil Weingarten einen erschreckend hohen Stimmenanteil von 20,7 \% für die AfD. Damit lag dieses Quartier 5,5 \% über dem Landesdurchschnitt. Bei der Bundestagswahl 2017 kam die AfD in Weingarten erneut auf einen Spitzenwert von 17,2 \%. Die AfD gewann landesweit 12,2\% der Stimmen und erzielte den größten Stimmenzuwachs in Baden-Württemberg von 6,9 \% (vgl. Stadt Freiburg 2016). Auf Vergleiche mit ähnlichen Quartieren in anderen Großstädten soll an dieser Stelle verzichtet werden. In Freiburg gibt es jedoch einen zweiten Stadtteil, der Weingarten in manchem gleicht; das ist die Hochhaussiedlung Landwasser. Hier gibt es analoge Entwicklungen (vgl. Stadt Freiburg 2016, 2018). Festzuhalten ist zumindest ein landes- und bundesweiter Trend zu einem Erstarken rechtspopulistischer Kräfte, das allerdings in sozial belasteten Quartieren noch eine besondere Ausprägung erhält.

Eine differenzierte Stimmenanalyse in Freiburg-Weingarten im Hinblick auf einzelne Stimmbezirke, die beispielsweise eine Korrelation mit den Partizipationsmodellen zuließe, war anhand des vorliegenden Materials leider nicht möglich und auch andere fundierte, öffentlich zugängliche Analysen und Reflexionen stehen leider nicht zur Verfügung. So lässt sich hier nur darüber spekulieren, wie es ohne die langjährigen Bemühungen der Gemeinwesenarbeit oder anderer zivilgesellschaftlicher Akteur*innen aussähe. 


\section{Widersprüchliche Wirksamkeiten trotz erfolgreicher Interventionen}

Die bisherigen erfolgreichen Interventionen durch die Gemeinwesenarbeit in Form von nachbarschaftlichen Begegnungen und Beteiligungsmodellen gehen mit einer Vielzahl von Interventionen zur Verbesserung der Lebensverhältnisse vor Ort einher. Aber sie spiegeln sich offensichtlich nicht unmittelbar im Wahlverhalten aller Bewohner*innen wider. Das wirft durchaus Fragen nach der Haltbarkeit der hohen Erwartungen beispielsweise an die Übertragbarkeit des Theoriemodells von John Dewey auf.

In der kritischen Reflexion stellen sich jedoch Fragen, ob nicht wesentliche Aspekte der deweyschen Theorie übersehen wurden. Eines fällt sofort auf: Dewey verweist immer wieder auf die Notwendigkeit umfassender Bildungsprozesse, die „in jedem einzelnen ein persönliches Interesse an sozialen Beziehungen und Kontrollen weckt und das zugleich die geistigen Gewohnheiten erzeugt, die soziale Veränderungen ermöglichen, ohne Chaos herbeizuführen“" (Dewey 1916: 105). Die Einsicht in demokratische Abläufe und Systeme wird nicht zufällig oder gar zwangsläufig in den Menschen geweckt, ebenso wenig die Fähigkeit, komplexe gesellschaftliche Prozesse in all ihren Widersprüchen und Verwerfungen so zu reflektieren, dass hieraus konstruktive Mitwirkungsambitionen erwachsen könnten. Nach Dewey spiele die Erziehung dabei eine entscheidende Rolle.

„Damit sie nicht impulsiv handelten, apathisch abseits ständen, sich gar bevormunden und indoktrinieren ließen, sollten alle Menschen außer einer hohen Allgemeinbildung auch - und vor allem aufgrund der wesenhaften Beziehung zwischen Demokratie und Wissenschaft - eine alltagstaugliche sozialwissenschaftliche Grundbildung erhalten“" (Knoll 2018: a.a.O.).

Eine solche Bildung kann in Quartieren wie beispielsweise Freiburg-Weingarten nicht vorausgesetzt werden. Hier lebt ein überdurchschnittlich hoher Anteil von Menschen mit verhältnismäßig niedrigen Bildungsabschlüssen. Nebenbei bemerkt ist auch bei Bürger*innen mit Hochschulabschluss nicht unbedingt zu erwarten, dass sie die politischen Prozesse hinreichend kritisch und differenziert interpretieren können. Professionskritisch soll an dieser Stelle aber auch in Erinnerung gebracht werden, dass seit den 1990er-Jahren des vergangenen Jahrhunderts in der Gemeinwesenarbeit gegenüber der Pädagogik und Erwachsenenbildung eine eher ablehnende Haltung eingenommen wurde. Nicht zuletzt die durch Wolfgang Hinte repräsentierte „Sozialraumorientierung“ plädierte dafür, „nicht die Menschen, sondern die Verhältnisse verändern“ (Hinte \& Threes 2007: 34) zu wollen. Das führte u. a. dazu, dass das Augenmerk in der Gemeinwesenarbeit zunehmend auf strukturelle und materielle Veränderungen in den Lebenslagen der Menschen gerichtet wurde. Bildungs- oder gar Persönlichkeitsentwicklungsprozesse wurden 
vielmals erst gar nicht thematisiert. Es soll dagegen die These erhoben werden, dass es entsprechend der dialektischen Verflechtungen von System und Individuum, Umwelt und Personen, Sozialraum und Lebenswelt etc. nachgerade unumgänglich erscheint, sowohl Verhalten als auch Verhältnisse verändern zu wollen. Das impliziert keineswegs paternalistische oder gar autoritäre Konzepte; vielmehr geht es immer auch um Angebote, Anregungen und die Befähigung von Eigenbildung und gemeinschaftlichem Lernen ganz im Sinne der Aufklärung, wonach Emanzipation als die Befreiung aus der selbst verschuldeten Unmündigkeit verstanden wird. Alle Bildungsansätze implizieren letztendlich aber doch Anregungen und Auseinandersetzungen zwischen aktuellen Erkenntnissen, Einstellungen und Deutungsmustern und möglichen, wenn nicht gar notwendigen alternativen Mustern und Konzepten. Voraussetzungen im deweyschen Modell wären vertrauensvolle Beziehungen auf Augenhöhe und die Offenheit und Bereitschaft, miteinander und voneinander zu lernen.

Ferner finden wir bei Dewey die Anregung, dass diese Demokratisierungsprozesse in der Nachbarschaft bzw. im Quartier immer auch wissenschaftlich begleitet und reflektiert werden sollten. Zwar sind die hauptamtlichen Fachkräfte und auch einige ehrenamtlich tätige Aktivist*innen durchaus wissenschaftlich ausgebildet und teilweise auch profiliert; das Gesamtkonzept kennt jedoch keine strukturelle Verortung wissenschaftlicher Begleitung und Forschung. Im Gegenteil: In einer konsequenten Anwendung der Handlungsprinzipien Lebensweltorientierung und Empowerment werden die Bewohner*innen als Expert*innen ihrer Lebensverhältnisse in entscheidenden Schlüsselfunktionen gesehen; eine unabhängige wissenschaftliche Expertise von außen wird eher als drohende „Kolonialisierung der Lebenswelt" empfunden. Auch wenn dies fachlich durchaus erst einmal nachvollziehbar ist, sollte doch darüber nachgedacht werden können, ob nicht gerade die bedenklichen Entwicklungen im Wahlverhalten der Stadtteilbewohner*innen durch eine unabhängige wissenschaftliche Expertise reflektiert einem Diskurs im Stadtteil zugänglich gemacht werden sollten.

Der Blick in die Geschichte der Gemeinwesenarbeit sollte der Inspiration dienen. Gleichzeitig hinterlässt gerade das Denkmodell von Dewey aber auch die Sorge der Überforderung. Kann es tatsächlich gelingen, in der Tradition von Jane Addams und John Dewey heute in den Quartieren mit besonderen Entwicklungsbedarfen unserer großen Städte solche Prozesse zu initiieren und nachhaltig zu verankern, die den enormen gesellschaftlichen Irritationen und Verwerfungen standhalten können?

John Dewey selbst verwies darauf, wie hoch die Messlatte gelegt wurde: „Das Ziel der Demokratie ist ein radikales Ziel. Denn es ist ein Ziel, das bisher noch zu keiner Zeit und in keinem Land adäquat verwirklicht worden ist" (Dewey 1937: 299). Für die Theorie wie für die Praxis der Gemeinwesenarbeit darf das 
nicht abschreckend wirken. Vielmehr gilt es, lang-, mittel- und kurzfristige Zielsetzungen so zu formulieren, dass die nächsten Etappen Schritt für Schritt erreichbar sind, ohne die hochgesteckten Leitziele aus den Augen zu verlieren.

Insbesondere aber gilt es, das Demokratieverständnis im Sinne von Dewey neu zu denken. So wichtig das Wahlverhalten auch sein mag: Demokratie meint mehr, als alle paar Jahre am Wahltag zu den Urnen zu gehen. Demokratie ist eine umfassende Lebensweise, in der möglichst viele Menschen gleichermaßen an sozialen, kulturellen und wirtschaftlichen Gütern teilhaben können und die wechselseitigen Beziehungen untereinander kommunikativ und fair regeln. Gemeinwesenarbeit in diesem Verständnis kann dann durchaus wertvolle Beiträge zur Demokratieentwicklung liefern.

\section{Literatur}

Alinsky, S. (1941). Community Analysis and Organization. American Journal of Sociology, 46(6): 797-808.

Bauchmüller, M. (2018): Diktatur gewinnt, Demokratie verliert. Abrufbar unter: https:// www.sueddeutsche.de/politik/bertelsmann-studie-demokratie-diktatur-1.3915984. Letzter Zugriff: 20.9.2018.

Bertelsmann Stiftung (2015): Der typische Nichtwähler kommt aus sozial schwachem Milieu. Abrufbar unter: https://www.bertelsmann-stiftung.de/de/presse/pressemitteilungen/pressemitteilung/pid/der-typische-nichtwaehler-kommt-aus-sozial-schwachemmilieu/. Letzter Zugriff: 20.9.2018.

Bochtler, A. (2011): Die meisten Mieter machen mit. Hohe Beteiligung bei den Sprecherratswahlen in Weingarten. In: Badischen Zeitung vom 25.10.2011. Abrufbar unter: http://www.badische-zeitung.de/freiburg-suedwest/die-meisten-mieter-machen-mit-50941202.html. Letzter Zugriff: 20.9.2018.

Bonhoeffer, D. (1985): Widerstand und Ergebung. Briefe und Aufzeichnungen aus der Haft. Herausgegeben von Eberhard Bethge. Gütersloh: TB Siebenstern.

Brox, Annette (2012): Bewohnerbeteiligung ernst gemacht. In: Neue Caritas, Heft 8: 21-25.

Bundesministerium für Arbeit und Soziales (2017): Lebenslagen in Deutschland - Fünfter Armuts- und Reichtumsbericht der Bundesregierung. Berlin.

Dewey, J. (1916): Democracy and Education. MW 9. Carbondale \& Edwardsville: Southern Illinois University Press.

Dewey, J. (1927): The public and its problems. New York: Henry Holt and Company.

Dewey, J. (1937): Democracy Is Radical. LW 11. Carbondale \& Edwardsville: Southern Illinois University Press.

Dewey, J. (1988): Essays, Reviews, Miscellany, and The Public and Its Problems. LW 2. Carbondale \& Edwardsville: Southern Illinois University Press.

Eribon, D. (2016): Rückkehr nach Reims. Berlin: Suhrkamp.

Forum Weingarten (1989): Satzung des Forums Weingarten vom 14.6.1989, Freiburg im Breisgau. 
Freire, P. (1973): Pädagogik der Unterdrückten. Bildung als Praxis der Freiheit. Reinbek bei Hamburg.

Gronemeyer, R. \& Bahr, H.-E. (Hg.) (1977): Nachbarschaft im Neubaublock. Empirische Untersuchungen zur Gemeinwesenarbeit, theoretische Studien zur Wohnsituation. Weinheim, Basel.

Habermas, J. (2013): Im Sog der Technokratie. Berlin: Suhrkamp.

Hausschild, J. (2016): Wahlverhalten. Warum rechts so verlockend ist. Abrufbar unter: https://www.spektrum.de/news/wer-waehlt-die-afd-und-warum/1423189. Letzter Zugriff: 20.9.2018.

Hinte, W. \& Tress, H. (Hrsg.) (2007): Sozialraumorientierung in der Jugendhilfe. Weinheim: Juventa Verlag.

Kant, I. (1784): Beantwortung der Frage: Was ist Aufklärung? Berlinische Monatsschrift 2: 481-494.

Klus, S. (2013): Die europäische Stadt unter Privatisierungsdruck. Konflikte um den Verkauf kommunaler Wohnungsbestände in Freiburg. Wiesbaden: Springer VS.

Knoll, M. (2018): Anders als gedacht. John Deweys Erziehung zur Demokratie. In: Zeitung für Pädagogik, Heft 5, zitiert nach http://www.mi-knoll.de/168401.html. Letzter Zugriff: 1.5.2018.

Merkel, W. (2013): Zukunft der Demokratie - Krise? Krise! In: Frankfurter Allgemeine online, 5.5.2013. Abrufbar unter: http://www.faz.net/aktuell/politik/die-gegenwart/zu kunft-der-demokratie-krise-krise-12173238.html. Letzter Zugriff: 20.9.2018.

Müller, W. C. (1988): Wie Helfen zum Beruf wurde: Eine Methodengeschichte der Sozialen Arbeit. Band I und II. Weinheim, Basel.

Offe C. (2003), Herausforderungen der Demokratie. Zur Integrations- und Leistungsfähigkeit. Frankfurt am Main, New York: Campus Verlag.

Stadt Freiburg (2016): Landtagswahl 2016, Wahlkreis 47. Abrufbar unter: https://fritz.freiburg.de/wahl/app/ltw2016.html. Letzter Zugriff: 20.9.2018.

Stadt Freiburg (2018): Ergebnis der Oberbürgermeisterwahl in Freiburg 22.4.2018. Abrufbar unter: https://fritz.freiburg.de/wahl/ob18.htm. Letzter Zugriff: 20.9.2018.

Staub-Bernasconi, S. (1995): Systemtheorie, soziale Probleme und Soziale Arbeit: lokal, national, international, oder: vom Ende der Bescheidenheit. Bern, Stuttgart, Wien.

Picht, W. (1913): Toynbee Hall und die englische Settlement-Bewegung. Tübingen: Mohr Verlag.

Weidenfeld, U. (2017): Regierung ohne Volk: Warum unser politisches System nicht mehr funktioniert. Berlin: Rowohlt Verlag.

Wendt, W. R. (1990): Geschichte der sozialen Arbeit. Stuttgart: Enke Verlag.

Open Access Dieses Kapitel wird unter der Creative Commons Namensnennung 4.0 International Lizenz (http://creativecommons.org/licenses/by/4.0/deed.de) veröffentlicht, welche die Nutzung, Vervielfältigung, Bearbeitung, Verbreitung und Wiedergabe in jeglichem Medium und Format erlaubt, sofern Sie den/die ursprünglichen Autor(en) und die Quelle ordnungsgemäß nennen, einen Link zur Creative Commons Lizenz beifügen und angeben, ob Änderungen vorgenommen wurden.

Die in diesem Kapitel enthaltenen Bilder und sonstiges Drittmaterial unterliegen ebenfalls der genannten Creative Commons Lizenz, sofern sich aus der Abbildungslegende nichts anderes ergibt. Sofern das betreffende Material nicht unter der genannten Creative Commons Lizenz steht und die betreffende Handlung nicht nach gesetzlichen Vorschriften erlaubt ist, ist für die oben aufgeführten Weiterverwendungen des Materials die Einwilligung des jeweiligen Rechteinhabers einzuholen. 